\title{
04. Relationship between depression, anxiety, and pain perception in diabetic neuropathy
}

Maria Nianiarou ${ }^{1, *}$, Zoi Gambopoulou², Dimitra Solomou ${ }^{3}$, Marianna Karathanou ${ }^{3}$, Dimitra Papadopoulou ${ }^{3}$, Evmorfia Stavropoulou ${ }^{2}$

${ }^{1}$ Outpatient Chronic Pain Department, KAT Hospital, Athens, Greece; ${ }^{2}$ Department of Anestesiology, Outpatient Chronic Pain Deepartment, KAT Hospital, Athens, Greece; ${ }^{3}$ Department of Anestesiology, KAT Hospital, Athens, Greece. *maryn0213@gmail.com

Introduction: Diabetic neuropathy is a common complication of diabetes, occurring in $25-50 \%$ of patients, with pain partaking in the clinical picture of about half of the patients. At the same time, there are many studies confirming the high prevalence of mental disorders in diabetes patients, and recent research signifies the bidirectional relationship that seems to exist. In this review, we examined the existing literature regarding the role of anxiety and depression in diabetic neuropathy.

Methods: Utilizing PubMed as our search engine, we performed a search of the existing literature of the last decade. The key-words used were "depression" "anxiety" "pain" and "diabetic neuropathy". This search led us to 189 results, and out of those 13 were found to correlate with our criteria and were used in this paper.

Results: From the analysis of the existing literature we cite studies concluding that people with depression are more susceptible to the development of diabetes. Moreover, anxiety and depression are correlated with complications and higher prevalence of painful neuropathy. In diabetic patients, symptoms of anxiety and depression are independently connected with more pain. At a pathophysiology level, disorders in the cellular function of the CNS, such as central sensitization and changes in neuroglia, are noticed both in neuropathic pain and depression.

Conclusions: This review highlights the connection between pain and depression in patients with diabetic neuropathy, with emphasis on the effect of anxiety and depression in neuropathic pain. With the association with the pathophysiological pathways and the clinical phenotype further researched, new goals in the prevention and the treatment of pain in diabetic neuropathy can be studied. 